\title{
Non-perturbative and self-consistent models of neutron stars in $R$-squared gravity
}

\author{
Stoytcho S. Yazadjiev, ${ }^{a, b}$ Daniela D. Doneva, ${ }^{a}$ \\ Kostas D. Kokkotas, ${ }^{a}$ Kalin V. Staykov ${ }^{b}$ \\ ${ }^{a}$ Theoretical Astrophysics, Eberhard-Karls University of Tübingen, Tübingen 72076, Ger- \\ many \\ ${ }^{b}$ Department of Theoretical Physics, Faculty of Physics, Sofia University, Sofia 1164, Bul- \\ garia \\ E-mail: yazad@phys.uni-sofia.bg, daniela.doneva@uni-tuebingen.de, \\ kostas.kokkotas@uni-tuebingen.de, kalin.v.staikov@gmail.com
}

\begin{abstract}
In the present paper we investigate non-perturbatively and self-consistently the structure of neutron stars in $R$-squared gravity by simultaneously solving the interior and exterior problem. The mass-radius relations are obtained for several equations of state and for wide range of the $R$-squared gravity parameter $a$. Even though the deviation from general relativity for nonzero values of $a$ can be large, they are still comparable with the variations due to different modern realistic equations of state. That is why the current observations of the neutron star masses and radii alone can not put constraints on the value of the parameter a. We also compare our results with those obtained within the perturbative method and we discuss the differences between them.
\end{abstract}

Keywords: modified gravity, neutron stars 


\section{Contents}

1 Introduction $\quad 1$

2 Basic equations 23

3 Numerical results $\quad 6$

4 Conclusion $\quad 10$

\section{Introduction}

General relativity (GR) is well-tested in the weak-field regime, whereas the strong-field regime remains essentially unexplored and unconstrained. Namely in the strong regime, GR is expected to break down and give way to a more complete theory of gravitation. Indeed, it is well-known that the quantum corrections in the strong field regime give rise to a modification of Einstein gravity - the renormalization at one loop demands that the Einstein-Hilbert action be supplemented with higher order terms [1]. On the other hand, the attempts to construct a unified theory of the interactions, naturally lead to scalar-tensor type generalizations of General Relativity and theories of gravity with Lagrangians containing various kinds of curvature corrections to the usual Einstein-Hilbert Lagrangian [2].

Besides the theoretical reasons, there are well-known observational facts that force us to go beyond the original Einstein theory and to study modified gravity theories. One of the most important discoveries in physics in the last two decades was the accelerated expansion of the Universe. The cause for accelerated expansion of the Universe is a mystery at present, and it is a great challenge for physics to solve this problem. There are, however, two general hypothesis for explaining the acceleration of the Universe. According to the first hypothesis, there exists a new kind of matter, called dark energy, which governs the accelerated expansion of the Universe [3]. Within the framework of this hypothesis the dark energy constitutes $73 \%$ of the total energy content of the Universe and it exhibits some rather unusual and strange properties such as negative pressure to density ratio.

The second hypothesis for the explanation of the accelerated expansion of the universe is that general relativity (i.e. Einstein equations) should be modified. In other words, instead of attributing the accelerated expansion to unknown constituents of the Universe with rather unusual and strange properties, one can attribute it to our lack of understanding of gravity. In particular, one may consider that the accelerated expansion indicates the break down of general relativity at cosmological and even at astrophysical scales. Ones of the most popular generalized gravitational theories which can give a possible explanation of the present cosmological observations are the $f(R)$ theories [4],[5],[6].

The $f(R)$ theories, as it is well-known, are modifications of GR in which the usual Einstein-Hilbert Lagrangian is replaced with an appropriately chosen function of the scalar curvature $R$. By their very construction, the $f(R)$ theories inevitably contain dimensionful parameters which, at present, should be found or constrained by the experiments and the observations. In general, these parameters could determine rather different scales (e.g. from scales typical for the compact objects to cosmological scales) and can be responsible for the strong and weak field regime of the theory. 
Neutron stars are natural laboratories for investigating the strong-field regime of gravity and for testing the alternative gravitational theories. That is why neutron stars in $f(R)$ theories are currently an active field of research and they were studied by many authors [7]-[16]. The study of neutron star structure in $f(R)$ theories also might allow us to impose constraints on the parameters responsible for the strong field regime. A serious problem in using the neutron stars for testing the alternative theories and imposing constraints on their parameters is the uncertainty in the equation of state (EOS). The EOS uncertainty does not allow for accurate parameter constraints when the deviations from GR are of the same order as the deviations induced by different EOS. Nevertheless, at present we have some observational constraints on mass-radius relation for neutron stars which can be used in some extent to eventually constrain the parameters responsible for the strong field regime of the alternative theories.

A drawback of most of the works on neutron stars in $f(R)$ gravity is the method of investigation adopted in them. They use a perturbative scheme in order to study the neutron stars in $f(R)$ theories. More precisely they consider $f(R)$ theories as a perturbation to GR. In our opinion the use of a perturbative method to investigate the strong field regime in $f(R)$ theories is not completely justified, and might lead to unphysical results. It is well known that even if in the weak field regime modified gravity is indistinguishable from general relativity, nonlinear phenomena may appear in the strong field regime with serious consequences for the structure and the properties of the compact objects [17]-[20].

Another drawback in most of the current studies of neutron stars in $f(R)$ theories is the fact that the interior and exterior problems are not solved simultaneously as the self-consistent approach requires. Instead, the exterior solution is imposed to be the Schwarzschild one which is equivalent to freeze the scalar degree of freedom outside the star in contradiction with the field equations. In this way the self-consistency of the whole problem is violated and this may lead (and leads) to artificial effects on the neutron star structure. It is also worth mentioning that some papers consider strong field regime parameters with wrong sign which means that the conditions for absence of tachyonic instabilities are not satisfied leading to new but nonphysical branches of neutron star solutions. For example, such non-physical solutions were obtained within the framework of the $R$-squared gravity defined by $f(R)=R+a R^{2}$. In addition to the physical solutions with $a>0$ in [9], new but non-physical solutions were also obtained there by considering the unphysical sector with $a<0$ plagued with a tachyonic instability and with a seemingly wrong asymptotic expansion.

The aim of this paper is to investigate non-perturbatively and self-consistently the neutron stars in one of the best known examples of $f(R)$ theories, namely the $R$-squared gravity $f(R)=R+a R^{2}$ by simultaneously solving the interior and the exterior problem. We also comment on the possible constraints on the parameter $a$ that can be imposed if the current observations of the neutron star mass and radius are taken into account.

The non-perturbative and self-consistent re-examination of neutron stars in $f(R)$ theories is important for several reasons. The comparison between the perturbative methods and the non-perturbative one is very instructive for the future studies. The constraints on the parameters of the $f(R)$ theories obtained via the perturbative methods may be misleading in confronting the theory with the experiments and observations. The non-perturbative method can cover much wider ranges of the parameters and the parameter constrains based on it are much more reliable. Moreover, the perturbative methods predicts the possible existence of qualitatively new stable neutron star branches in comparison with GR - a rather drastic prediction in its own. So, non-perturbative confirmation or rejection of the possible existence of 
new stable neutron star branches is needed.

As we shall see below the non-perturbative method gives results that are different from the ones obtained by the perturbative method.

The paper is organized as follows. In Section 2 we give the basic framework of the problem and the reduced field equations that will be solved numerically. In Section 3 we present and discuss the results for neutron stars with realistic equations of state in nonperturbative $R$-squared gravity. We also compare our results with those obtained via the perturbative approach. We end the paper with discussion and conclusions.

\section{Basic equations}

The action of the $f(R)$ theories is given by

$$
S=\frac{1}{16 \pi G} \int d^{4} x \sqrt{-g} f(R)+S_{\text {matter }}\left(g_{\mu \nu}, \chi\right),
$$

where $R$ is the scalar curvature with respect to the spacetime metric $g_{\mu \nu}$ and $S_{\text {matter }}$ is the action of the matter fields denoted by $\chi$. The viable $f(R)$ theories have to be free of tachyonic instabilities and the appearance of ghosts which require [4],[5]

$$
\frac{d^{2} f}{d R^{2}} \geq 0, \quad \frac{d f}{d R}>0
$$

respectively.

It is well-known that the $f(R)$ theories are equivalent to the Brans-Dicke scalar-tensor theory with $\omega_{B D}=0$ and with a potential for the scalar field. This can be easily demonstrated by considering a new field $\psi$ and the dynamically equivalent action

$$
S=\frac{1}{16 \pi G} \int d^{4} x \sqrt{-g}\left[f(\psi)+f^{\prime}(\psi)(R-\psi)\right]+S_{\text {matter }}\left(g_{\mu \nu}, \chi\right) .
$$

Varying then with respect to $\psi$ one obtains $f^{\prime \prime}(\psi)(R-\psi)=0$ which, provided that $f^{\prime \prime}(\psi) \neq 0$, gives $\psi=R$. This substituted back in (2.3) indeed recovers (2.1). Introducing the new field $\Phi=f^{\prime}(\psi)$ and defining the potential $U(\Phi)$ via

$$
U(\Phi)=\psi(\Phi) f^{\prime}(\Psi(\Phi))-f(\psi(\Phi))
$$

our action (2.3) takes exactly the form of the action of Brans-Dicke theory in Jordan frame with a potential for the scalar field, namely

$$
S=\frac{1}{16 \pi G} \int d^{4} x \sqrt{-g}[\Phi R-U(\Phi)]+S_{\text {matter }}\left(g_{\mu \nu}, \chi\right)
$$

Especially, in the case of $R$-squared gravity $\left(f(R)=R+a R^{2}\right)$, the Brans-Dicke potential is given by 


$$
U(\Phi)=\frac{1}{4 a}(\Phi-1)^{2}
$$

which corresponds to a massive scalar field with a mass $m_{\Phi}=\frac{1}{\sqrt{6 a}}$. Here we consider only non-negative values for the parameter $a$ which obey the condition $\frac{d^{2} f}{d R^{2}} \geq 0$.

From a mathematical and numerical point of view, as in the general scalar-tensor theories, it is more convenient to study the field equations in the so-called Einstein frame. The Einstein frame is defined by introducing the new scalar field $\varphi$ and the new metric $g_{\mu \nu}^{*}$ given by

$$
\begin{aligned}
& \varphi=\frac{\sqrt{3}}{2} \ln \Phi, \\
& g_{\mu \nu}^{*}=\Phi g_{\mu \nu}=A^{-2}(\varphi) g_{\mu \nu}
\end{aligned}
$$

with $A^{2}(\varphi)=\Phi^{-1}(\varphi)=e^{-\frac{2}{\sqrt{3}} \varphi}$. The Einstein frame action then takes the form

$$
S=\frac{1}{16 \pi G} \int d^{4} x \sqrt{-g^{*}}\left[R^{*}-2 g^{* \mu \nu} \partial_{\mu} \varphi \partial_{\nu} \varphi-V(\varphi)\right]+S_{\text {matter }}\left(e^{-\frac{2}{\sqrt{3}} \varphi} g_{\mu \nu}^{*}, \chi\right),
$$

where $R^{*}$ is the Ricci scalar curvature with respect to the Einstein frame metric $g_{\mu \nu}^{*}$ and $V(\varphi)=A^{4}(\varphi) U(\Phi(\varphi))$. For the $R$-squared gravity the explicit form of the potential in the Einstein frame is

$$
V(\varphi)=\frac{1}{4 a}\left(1-e^{-\frac{2 \varphi}{\sqrt{3}}}\right)^{2}
$$

The expense for simplifying the action in Einstein frame (and the field equations as a consequence) is the appearance of direct interaction between the matter fields and the scalar field $\varphi$ in this frame. Taking variation with respect to the metric $g_{\mu \nu}^{*}$ and the scalar field $\varphi$, we find the field equations in the Einstein frame

$$
\begin{aligned}
& G_{\mu \nu}^{*}=8 \pi G T_{\mu \nu}^{*}+2 \partial_{\mu} \varphi \partial_{\nu} \varphi-g_{\mu \nu}^{*} g^{* \alpha \beta} \partial_{\alpha} \varphi \partial_{\beta} \varphi-\frac{1}{2} V(\varphi) g_{\mu \nu}^{*} \\
& \nabla_{\mu}^{*} \nabla^{* \mu} \varphi-\frac{1}{4} \frac{d V(\varphi)}{d \varphi}=-4 \pi G \alpha(\varphi) T^{*}
\end{aligned}
$$

where

$$
\alpha(\varphi)=\frac{d \ln A(\varphi)}{d \varphi}=-\frac{1}{\sqrt{3}}
$$

The Einstein frame energy-momentum tensor $T_{\mu \nu}^{*}$ is related to the Jordan frame one $T_{\mu \nu}$ via $T_{\mu \nu}^{*}=A^{2}(\varphi) T_{\mu \nu}$. In the case of a perfect fluid, the energy density, the pressure and the 4 -velocity in the two frames are related via the formulae 


$$
\rho_{*}=A^{4}(\varphi) \rho, \quad p_{*}=A^{4}(\varphi) p, \quad u_{\mu}^{*}=A^{-1}(\varphi) u_{\mu} .
$$

The contracted Bianchi identities give the following conservation law for the Einstein frame energy-momentum tensor

$$
\nabla_{\mu}^{*} T_{\nu}^{* \mu}=\alpha(\varphi) T^{*} \nabla_{\nu}^{*} \varphi
$$

The next step is to consider a static and spherically symmetric spacetime described by the Einstein frame metric

$$
d s_{*}^{2}=-e^{2 \phi(r)} d t^{2}+e^{2 \Lambda(r)} d r^{2}+r^{2}\left(d \theta^{2}+\sin ^{2} \theta d \vartheta^{2}\right) .
$$

Since the purpose of the present paper is to study the structure of neutron stars in $f(R)$ gravity, we consider the matter source to be a perfect fluid. We also require the perfect fluid and the scalar field to respect the staticity and the spherical symmetry. With these conditions imposed, the dimensionally reduced field equations are

$$
\begin{aligned}
& \frac{1}{r^{2}} \frac{d}{d r}\left[r\left(1-e^{-2 \Lambda}\right)\right]=8 \pi G A^{4}(\varphi) \rho+e^{-2 \Lambda}\left(\frac{d \varphi}{d r}\right)^{2}+\frac{1}{2} V(\varphi) \\
& \frac{2}{r} e^{-2 \Lambda} \frac{d \phi}{d r}-\frac{1}{r^{2}}\left(1-e^{-2 \Lambda}\right)=8 \pi G A^{4}(\varphi) p+e^{-2 \Lambda}\left(\frac{d \varphi}{d r}\right)^{2}-\frac{1}{2} V(\varphi), \\
& \frac{d^{2} \varphi}{d r^{2}}+\left(\frac{d \phi}{d r}-\frac{d \Lambda}{d r}+\frac{2}{r}\right) \frac{d \varphi}{d r}=4 \pi G \alpha(\varphi) A^{4}(\varphi)(\rho-3 p) e^{2 \Lambda}+\frac{1}{4} \frac{d V(\varphi)}{d \varphi} e^{2 \Lambda} \\
& \frac{d p}{d r}=-(\rho+p)\left(\frac{d \phi}{d r}+\alpha(\varphi) \frac{d \varphi}{d r}\right)
\end{aligned}
$$

and they describe the interior structure, i.e. the spacetime metric, the energy density, pressure and scalar field inside the neutron star. Note that in the above system we used (2.14) and we substituted $\rho_{*}$ and $p_{*}$ with $A^{4}(\varphi) \rho$ and $A^{4}(\varphi) p$, respectively.

The equations describing the spacetime metric and the scalar field outside the neutron star are obtained from the above system by formally putting $\rho=p=0$. In addition to our systems of differential equations for the interior and the exterior of the neutron star, we should give the equation of state (EOS) for the neutron star matter $p=p(\rho)$ and impose the boundary conditions.

As we have already discussed in the introduction, we solve the interior and the exterior problem simultaneously with the following natural Einstein frame boundary conditions in the center of the star

$$
\rho(0)=\rho_{c}, \quad \Lambda(0)=0, \quad \frac{d \varphi}{d r}(0)=0,
$$

and at infinity

$$
\lim _{r \rightarrow \infty} \phi(r)=0, \quad \lim _{r \rightarrow \infty} \varphi(r)=0 .
$$


The coordinate radius $r_{S}$ of the star is determined by the condition

$$
p\left(r_{S}\right)=0 .
$$

Some comments on the boundary conditions are in order. The condition $\frac{d \varphi}{d r}(0)=0$ ensures the regularity of the scalar field $\varphi$, and in turn the regularity of $\Phi$, at the center $r=0$. The regularity of the Einstein frame geometry at the center requires $\Lambda(0)=0$. Since the Jordan and the Einstein frame metrics are conformally related (via nonsingular conformal factor) this condition also insures the regularity of the Jordan frame geometry at the center of the star. The boundary conditions at infinity are related to the fact that we consider neutron stars in asymptotically flat spacetime*. The asymptotic flatness requires $\lim _{r \rightarrow \infty} V(\varphi(r))=0$ which gives $\lim _{r \rightarrow \infty} \varphi(r)=0$ or equivalently $\lim _{r \rightarrow \infty} \Phi(r)=1$. Note that the conditions (2.22) ensure the asymptotic flatness in both Einstein and Jordan frame.

Although the coordinate radius of the star is determined via (2.23), the physical radius of the star as measured in the physical Jordan frame is given by

$$
R_{S}=A\left[\varphi\left(r_{S}\right)\right] r_{S}
$$

Concerning the mass of the neutron star, it can be found from the asymptotic expansion of the physical Jordan frame metric. However, especially for the case of $R$-squared gravity, the scalar field $\Phi$ (respectively $\varphi$ ) has a finite range, i.e. it drops off exponentially at infinity and this shows that the Jordan and the Einstein frame masses coincide.

In the numerical results, presented in the next section, we use the dimensionless parameter

$$
a \rightarrow \frac{a}{R_{0}^{2}}
$$

where $R_{0}=1.47664 \mathrm{~km}$ which corresponds to one solar mass.

\section{$3 \quad$ Numerical results}

The field equations (2.17)-(2.20) together with the boundary conditions (2.21) and (2.22) are solved numerically using a shooting method. Additional complications come from the fact that the presence of a nontrivial potential of the form (2.10) makes the system of differential equations stiff, with increasing stiffness as $a$ decreases. This requires refinement of the numerical algorithm and a close control of the shooting procedure.

Below we present in detail the obtained results. We use four realistic EOS with distinct properties in order to show the possible deviations from general relativity more thoroughly. The equations of state are SLy4 [21], APR4 [22], FPS [24] and L [23] $]^{\dagger}$. EOS SLy4 and APR4 are both modern realistic equations of state that fulfill all of the observational constrains on the neutron star mass and radius [26]-[30]. EOS FPS on the other hand is softer and its maximum mass does not reach the two solar mass barrier [29, 30]. Nevertheless we examined

\footnotetext{
${ }^{*}$ The assumption for asymptotically flat spacetime is completely justified for local astrophysical systems of size of $1 \mathrm{AU}$ or less, compared to the cosmological scales on the order of $10^{26} \mathrm{~m}$.

${ }^{\dagger}$ For EOS APR4 we used the piecewise polytropic approximation given in [25].
} 
it because, as we will show below, $f(R)$ gravity could alter considerably the maximum mass of neutron stars and eventually reconcile an EOS with the observations. EOS L is one of the stiffest proposed realistic EOS and even though it leads to somewhat larger radii than the current observational constraints, we will consider it as a limiting case in stiffness.

The mass of radius relations for the four realistic EOS are given in Figs. 1 and 2. The current observational constraints on the neutron star mass and radius, given in [27, 29], are shown as shaded regions on the graphs. Lines with different styles and colors in every figure correspond to different values of the parameter $a$ ranging from $a=0.3$ to $a=10^{4 \ddagger}$. As one can see, for all of the EOS, $a=0.3$ leads to models that are almost indistinguishable from general relativity especially for masses above one solar mass and for $a<0.3$ the neutron star solutions get closer and closer to the general relativistic ones. On the other hand when $a \rightarrow \infty$ the neutron star mass and radius saturates to certain values for a fixed central energy density and EOS, and $a=10^{4}$ gives us nearly the maximum possible deviation from the pure Einstein theory. The reason for this behavior is the following. The increase of $a$ is equivalent to decreasing the scalar-field mass: when $a \rightarrow \infty$ the mass of the scalar field vanishes and when $a \rightarrow 0$ the mass goes to infinity. Loosely speaking the nonzero mass suppresses the scalar field exponentially, and in general larger masses correspond to smaller values of the scalar field. That is why models with smaller $a$ are closer to general relativity, which is recovered in the limit $a \rightarrow 0$. When $a$ increases, the mass of the scalar field decreases and thus the scalar field can reach larger values. Consequently the deviations from general relativity also increase. The case $a \rightarrow \infty$ corresponds to $\omega_{B D}=0$ Brans-Dicke scalar-tensor theory with zero potential of the scalar field and represents the maximum possible difference between the considered $f(R)$ theory of gravity and general relativity.

A common feature for all of the realistic EOS is the following - for larger neutron star masses the presence of nontrivial scalar field leads to an increase of the neutron star radius. For smaller neutron star masses on the other hand the radius decreases. This behavior is qualitatively different from the perturbative results presented in [9], where for large masses the radius decreases and for small masses it increases compared to Einstein theory.

For all of the considered equations of state the maximum mass reaches up to approximately $10 \%$ larger values in the limit $a \rightarrow \infty$ compared with general relativity. This fact can help us for example to reconcile EOS FPS with the observation. In the general relativistic case its maximum mass is significantly below two solar masses, but for large values of the parameter $a$ the two solar mass barrier is reached. A similar observation can be made for EOS SLy4 that barely reaches two solar masses in the pure Einstein theory, but when $a$ increases the maximum mass also increases and the mass constraint is easily satisfied. On the other hand the maximum mass of the neutron star sequences can decrease for small values of $a$ (typically for $a<2$ ). But this decrease of the mass is very small - it does not exceed $1 \%$ for all of the realistic EOS.

As a whole the results in the present paper lead us to the idea that the differences between the $R$-squared gravity and general relativity are comparable with the uncertainties in the nuclear matter equations of state. That is why the current observations of the neutron star masses and radii alone can not put constraints on the value of the parameters a, unless the equation of state is better constrained in the future.

\footnotetext{
${ }^{\ddagger}$ We studied systematically the whole range from $a=0.02$ to $a=10^{5}$, but we use a narrower range of $a$ for a better representation of the data. The models with $a<0.3$ and $a>10^{4}$ are very close to the cases of $a=0.3$ and $a=10^{4}$ respectively.
} 

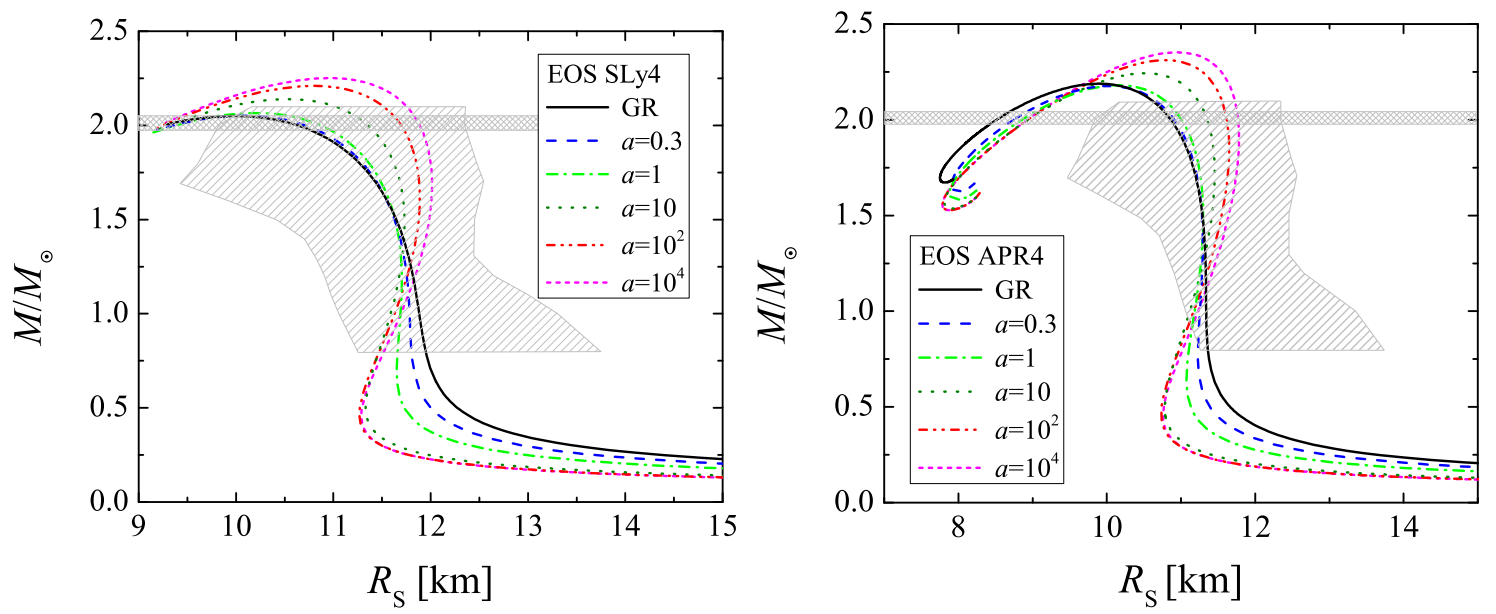

Figure 1. The mass of radius relation for EOS SLy4 (left panel) and APR4 (right panel). Different styles and colors of the curves correspond to different values of the parameter $a$. The current observational constrains are shown as shaded regions.
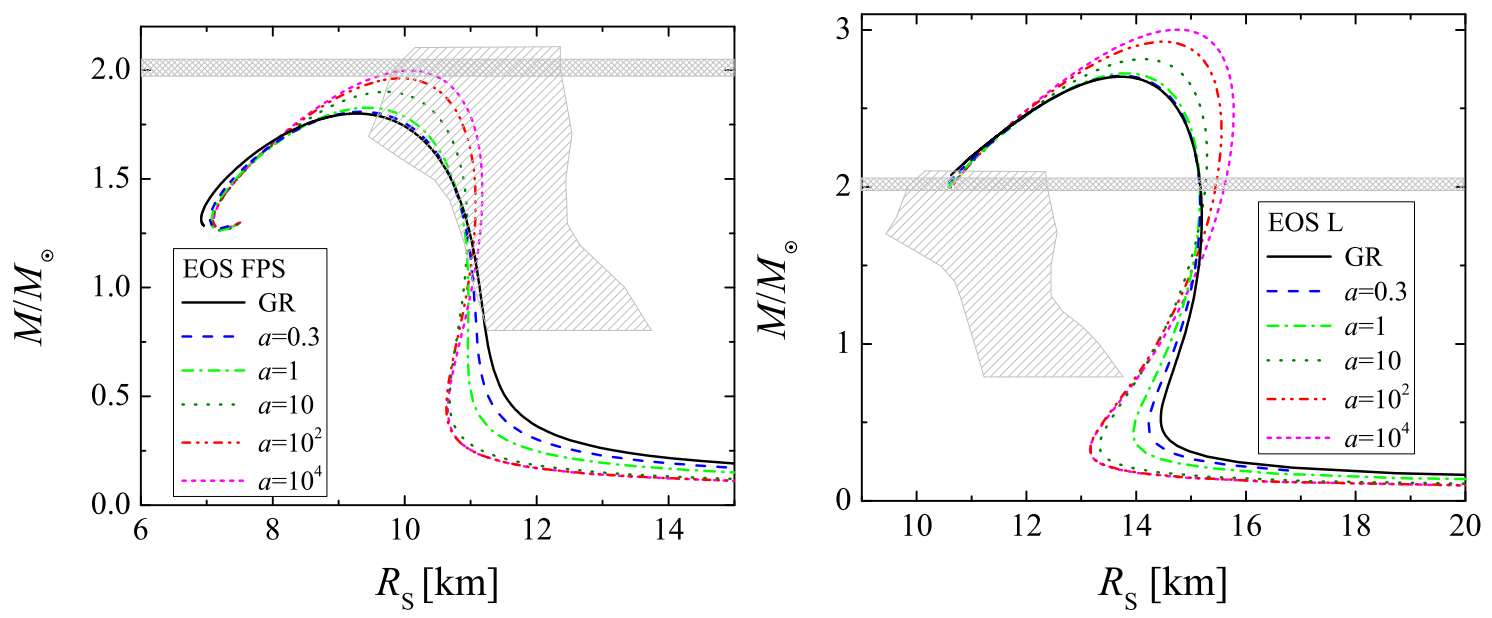

Figure 2. The mass of radius relation for EOS FPS (left panel) and L (right panel). Different styles and colors of the curves correspond to different values of the parameter $a$. The current observational constrains are shown as shaded regions.

It is interesting to examine more thoroughly how does the maximum mass changes when we vary the parameter $a$ and to compare our results with the ones obtained by the perturbative approach in [9]. In Fig. 3 we have plotted the maximum mass $M_{\max }$ as a function of $a$ for EOS SLy4 and APR4. In the figures the general relativistic case is presented as $a=0$. The behavior of the plotted dependences is characteristic for all of the studied EOS. When $a \rightarrow 0$ the maximum mass tends to the corresponding value in general relativity for a particular EOS. With the increase of $a, M_{\max }$ first decreases and after reaching a minimum it starts to increase monotonically. The maximum value of $M_{\max }$ is reached for $a \rightarrow \infty$ and it is up to approximately $10 \%$ larger than the pure Einstein theory depending on the EOS. The minimum is typically reached for $a<1$ and $M_{\max }$ deviates less than $1 \%$ compared with pure general relativity. For the considered realistic equations of state the radius of the corresponding maximum mass models $R_{\min }$ is a monotonically increasing function of $a$. 

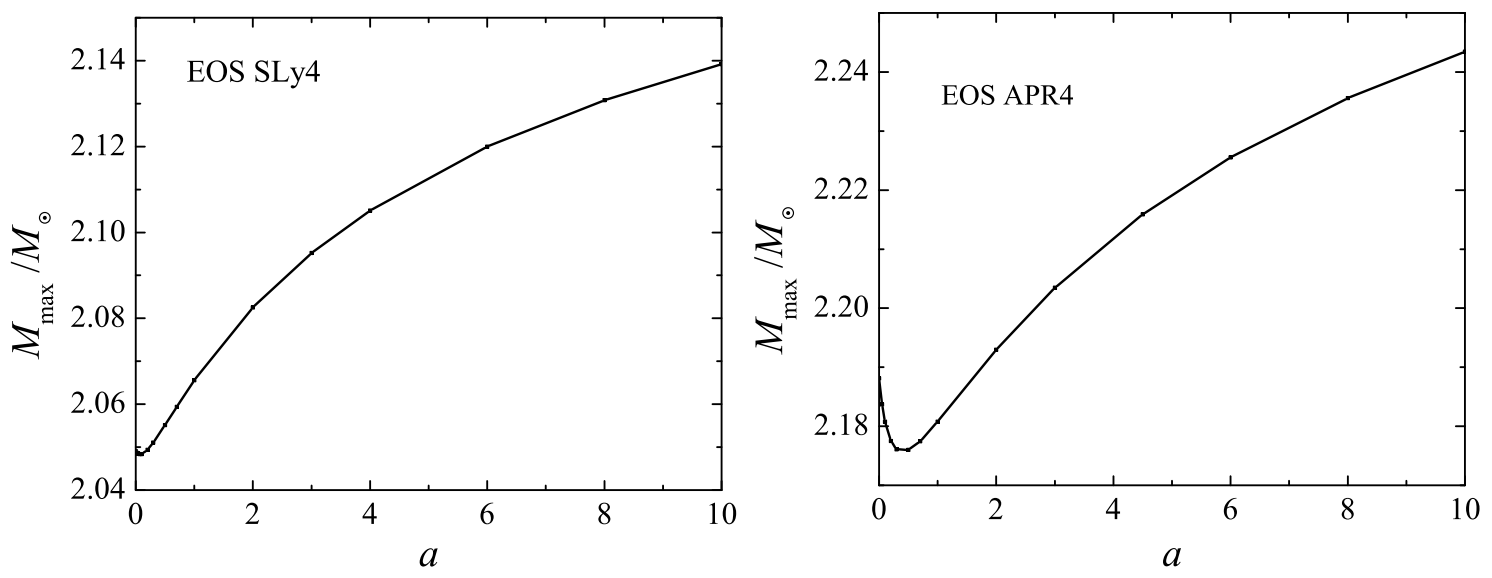

Figure 3. The maximum mass as a function of the parameter $a$ for EOS SLy4 (left panel) and APR4 (right panel). In the limit $a \rightarrow \infty, M_{\max }=2.25 M_{\odot}$ for EOS SLy4 and $M_{\max }=2.36 M_{\odot}$ for EOS APR4.

The decrease of $M_{\max }$ for small values of $a$ was also observed in [9], but the subsequent increase of the maximum mass was not reported there for any of the realistic EOS. In general there are noticeable quantitative differences between our results and those of [9]. The most important is that the decrease of the maximum mass observed in [9] is much stronger compared to the non-perturbative approach. As a consequence constraints on $a$ were obtained in [9] after a comparison with observation (more precisely they require that the maximum mass of the modern realistic equations of state should not fall below approximately two solar masses). Such constraints are obviously not possible in the non-perturbative approach where the decrease of the mass is very small and the deviations from general relativity are comparable with the deviations coming from the use of different modern realistic equations of state.

In order to be more precise we also made a systematic comparison with the results for a specific polytropic equation of state given in [9]. The value of the polytropic index used there is $\Gamma=9 / 5$. The mass $M_{\max }$ and radius $R_{\min }$ of the maximum mass models as a function of $a$ are presented in Figs. 4 and 5 for this polytropic EOS. The qualitative behavior of $M_{\max }$ and $R_{\min }$ is similar to the results in [9] - when we increase $a$ the values of $M_{\max }$ and $R_{\min }$ first decrease and after reaching a minimum they start to increase. Also the minima of $M_{\max }$ is at similar values of the parameter $a$ compared to the results in [9]. But the quantitative differences with the corresponding figures in [9] are significant. For example the minimum of $M_{\max }$ is considerably deeper in [9] compared to our results. The minimum of $R_{\min }$ on the other hand is located at much larger values of $a$ in our case and it reaches considerably smaller values of $R_{\min }$ compared to the perturbative approach.

It is also worth confronting the non-perturbative and self-consistent approach with the perturbative approach in their description of the local internal structure of the neutron stars. Within the perturbative and non-self-consistent approach it was found in [12] that for the equation of state SLy there are regions inside the star where the local Jordan frame mass decreases with the radial coordinate in a contra-intuitive way. For the same equation of state and the same value of the parameter $a$ no such behaviour is observed in the non-perturbative approach. The same holds also for the other EOS.

Another fact showing that the perturbative approach is not completely reliable concerns 

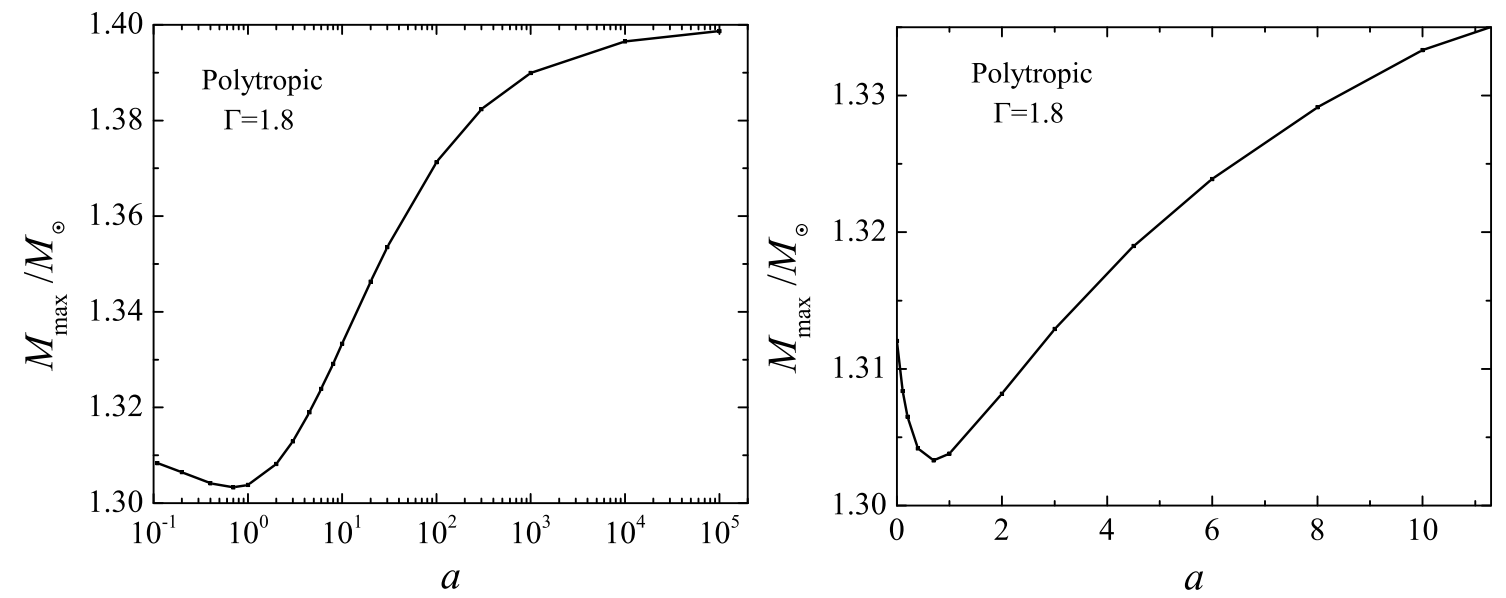

Figure 4. The maximum mass as a function the parameter $a$ for polytropic EOS with index $\Gamma=1.8$. The right figure is a magnification in a non-logarithmic scale.
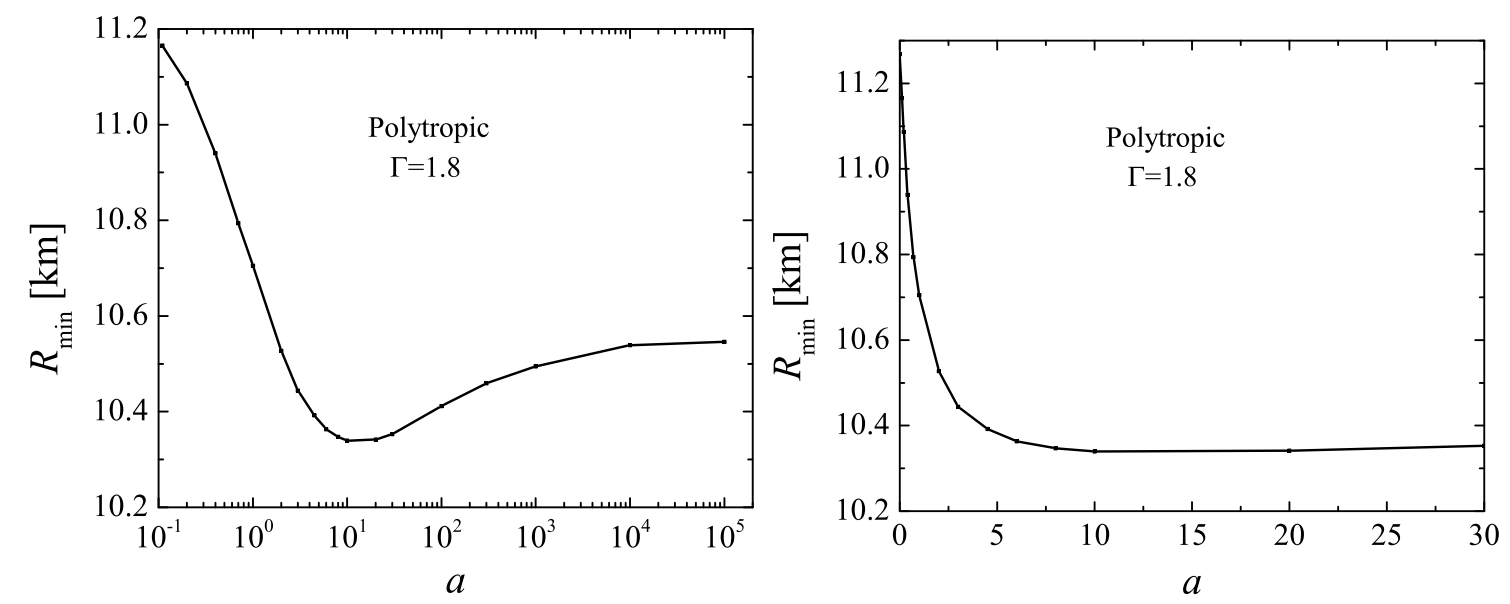

Figure 5. The minimum radius as a function the parameter $a$ for polytropic EOS with index $\Gamma=1.8$. The right figure is a magnification in a non-logarithmic scale.

the value of the "correction" $a R$ in the Lagrangian $f(R)=R(1+a R)$ and is the following. For all EOS and for all values of the parameter $a$ ranging from $a=2 \cdot 10^{-2}$ to $a=10^{5}$ we found that $|a R|$ does not exceed the value $10^{-1}$ anywhere throughout the star. Although $|a R|<10^{-1}$ is in the range where the perturbative approach is expected to work well, the results that it gives, as we saw above, can be rather different from those obtained via the non-perturbative and self-consistent approach.

\section{Conclusion}

In the present paper we studied models of neutron stars in $R$-squared gravity using a nonperturbative and self-consistent approach. We used the fact that the $f(R)$ theories of gravity are mathematically equivalent to a particular class of scalar-tensor theories with nonzero potential of the scalar field. Within this framework we constructed numerical solutions describing neutron stars with different realistic equations of state. A wide range of the parameter $a$ in the $R$-squared gravity was covered that is not possible when using the perturbative 
approach. We had two main goals - to study the possible deviations from general relativity and to compare our results with the widely used perturbative approach to $f(R)$ gravity in order to study the possible nonlinear effects.

Our results show that the differences in the neutron star properties induces by the $R$ squared theory of gravity can be considerable. For example, the maximum mass of neutron stars for a specific realistic equation of state can increase by approximately $10 \%$ depending on the equation of state. Thus for example equations of state which do not reach the two solar mass barrier in general relativity can be reconciled with the observation if we employ $f(R)$ theories of gravity. The neutron star radii also changes significantly - in general for large masses the radius increases whereas for small masses it decreases compared to Einstein theory. But we should note that even though the deviations from general relativity can be large, these deviations are still comparable with the uncertainties in the nuclear matter equation of state. Therefore the current observations of neutron star masses and radii alone can not put constraints on the free parameter in the $R$-squared theory. But if the nuclear equation of state is better constrained in the future, the current investigations would help us in constraining $f(R)$ theories of gravity via neutron star observations.

We addressed also in detail the comparison between the non-perturbative and selfconsistent approach from one side and the perurbative approach from the other. It turns out that the two approaches lead to both qualitative and quantitative different results, as it can be seen from the graphs presented in the present paper and those in [7] and [9]. The most significant difference is the behaviour of the maximum neutron star mass as a function of the parameter $a$ in the physical sector $a>0$. In both the perturbative and non-perturbative approach a decrease of the maximum mass is observed for small values of the parameter $a$, but the quantitative differences reach large values. In the non-perturbative approach, the decrease is almost negligible - below $1 \%$ for all of the realistic EOS. In contrast the results in [9] show a much stronger decrease of the maximum mass. Taking into account that the maximum mass of the modern realistic equations of state should not fall below approximately two solar masses, constraints on the parameter $a$ were obtained in [9], namely $a \lesssim 10^{6} \mathrm{~m}^{2}$. It is obviously not possible to derive such constraints in the non-perturbative approach were the decrease of the mass is very small and the deviations from general relativity are comparable to the deviations coming from the use of different modern realistic equations of state. Also in the non-perturbative approach a considerable increase (up to approximately 10\%) of the maximum mass is observed for large values of $a$ and for all of the considered EOS.

The behavior of the neutron star radius is also different - in the non-perturbative approach the models with larger masses have larger radii compared to general relativity and the models with smaller masses have smaller radii. This behaviour is exactly opposite to the perturbative models presented in [9]. In addition, new and potentially stable branches of solutions were not found after the maximum mass for neither of the considered EOS nor values of $a$.

Concerning the local internal structure of the stars in $f(R)$ gravity, the local Jordan frame mass increases with the radial coordinate for all of the considered equations of state, including SLy4, in contrast to the observed decrease of the mass with the radial coordinate found using a perturbative approach in [12].

Concluding, the perturbative approach does not seem able to provide reliable results for the study of the strong field regime of $f(R)$ theories and for confronting it with the observations.

In this situation, it seems that the tightest constraint on the parameter $a$ of the $R$ - 
squared gravity is imposed by the results from Gravity Probe B experiment, namely $a \lesssim$ $5 \times 10^{11} \mathrm{~m}^{2}$ [31]. In terms of the scalar field mass $m_{\Phi}$ the constraint corresponds to $m_{\phi} \gtrsim$ $10^{-13} \mathrm{eV} / \mathrm{c}^{2}$ where $c$ is the speed of light. However, this constraint is based on the weak field regime and it can change for more general $f(R)$ theories.

A natural generalization of the present work is to extend the non-perturbative and self-consistent approach to more general $f(R)$ theories. This is numerically considerably more challenging task and we hope to address the problem in the near future. Some of our preliminary studies show that the nonperturbative approach gives in the general case results different from the perturbative one.

\section{Acknowledgments}

D. D. would like to thank the Alexander von Humboldt Foundation for support. K.K. and S.Y. would like to thank the Research Group Linkage Programme of the Alexander von Humboldt Foundation for the support and S.Y. would like to thank the Institute for Theoretical Astrophysics Tuebingen for its kind hospitality. The support by the Bulgarian National Science Fund under Grant No. DMU-03/6, by the German Science Foundation (DFG) via SFB/TR7, and the networking support by the COST Action MP1304 is gratefully acknowledged.

\section{References}

[1] N.D. Birrell and P.C.W. Davies, Quantum fields in curved space, Cambridge University Press, Cambridge (1982)

[2] T. Clifton, P. G. Ferreira, A. Padilla and C. Skordis, Phys. Rept. 513 (2012) 1.

[3] A. G. Riess et al. [Supernova Search Team Collaboration], Astron. J. 116 (1998) 1009; S. Perlmutter et al. [Supernova Cosmology Project Collaboration], Astrophys. J. 517 (1999) 565; P. Astier et al. [The SNLS Collaboration], Astron. Astrophys. 447 (2006) 31; A. G. Riess et al. [Supernova Search Team Collaboration], Astrophys. J. 607 (2004) 665; A. G. Riess et al., Astrophys. J. 659 (2007) 98; N. Spergel et al. [WMAP Collaboration], Astrophys. J. Suppl. 170 (2007) 377; M. Kowalski et al. [Supernova Cosmology Project Collaboration], Astrophys. J. 686 (2008) 749; E. Komatsu et al. [WMAP Collaboration], Astrophys. J. Suppl. 180 (2009) 330.

[4] T. P. Sotiriou and V. Faraoni, Rev. Mod. Phys. 82 (2010) 451.

[5] A. De Felice and S. Tsujikawa, Living Rev. Rel. 13 (2010) 3.

[6] S. Nojiri and S. Odintsov, Phys. Rept. 505 (2011) 59.

[7] A. Cooney, S. DeDeo and D. Psaltis, Phys. Rev. D 82 (2010) 064033.

[8] E. Babichev and D. Langlois, Phys. Rev. D 81, (2010) 124051.

[9] A. S. Arapoglu, C. Deliduman and K. Y. Eksi, JCAP 1107 (2011) 020.

[10] L. Jaime, L. Patino and M. Salgado, Phys. Rev. D83 (2011) 024039.

[11] E. Santos, Astrophys. Space Sci. 341 (2012) 411.

[12] M. Orellana, F. Garcia, F. Pannia and G. Romero, Gen. Rel. Grav. 45, 4 (2013) 771.

[13] H. Alavirad and J. Weller, Phys. Rev. D88 (2013) 124034.

[14] A. Astashenok, S. Capozziello and S. Odintsov, JCAP 1312 (2013) 040.

[15] A. Ganguly, R. Gannouji, R. Goswami and S. Ray, arXiv:1309.3279 [gr-qc] 
[16] A. Astashenok, S. Capozziello, and S. Odintsov, arXiv:1401.4546 [gr-qc]

[17] T. Damour and G. Esposito-Farese, Physical Review Letters 70 (1993) 2220.

[18] I. Stefanov, S. Yazadjiev and M. Todorov, Mod. Phys. Lett. A23 (2008) 2915.

[19] D. Doneva, S. Yazadjiev, K. Kokkotas and I. Stefanov, Phys. Rev. D82 (2010) 064030.

[20] D. Doneva, S. Yazadjiev, N. Stergioulas and K. Kokkotas, Phys. Rev D88 (2013) 084060.

[21] F. Douchin and P. Haensel, Astron. Astrophys. 380 (2001) 151.

[22] A. Akmal, V. R. Pandharipande, and D. G. Ravenhall, Phys. Rev. C58 (1998) 1804.

[23] V. Pandharipande, D. Pines and R. A. Smith, ApJ 208 (1976) 550 .

[24] B. Friedman and V. R. Pandharipande, Nucl. Phys. A361 (1981) 502.

[25] J. S. Read, B. D. Lackey, B. J. Owen, and J. L. Friedman, Phys. Rev. D79 (2009) 124032.

[26] J. M. Lattimer, Annu. Rev. Nucl. Part. Sci. 62 (2012) 485.

[27] A. W. Steiner, J. M. Lattimer and E. F. Brown, ApJ 722 (2010) 33.

[28] F. Özel, Reports on Progress in Physics 76 (2013) 016901.

[29] John Antoniadis et al., Science 340 (20130) 6131.

[30] P. B. Demorest, T. Pennucci, S. M. Ransom, M. S. E. Roberts and J. W. T. Hessels, Nature 467 (2010) 1081.

[31] J. Näf and P. Jetzer, Phys. Rev. D81, (2010) 104003. 